BMJ Paediatrics Open

\title{
Knowledge and attitudes of university health students in the Pacific Islands towards childhood hearing loss and hearing services: a Samoan survey study protocol
}

\author{
Annette Kaspar (D) , ${ }^{1,2}$ Sione Pifeleti, ${ }^{1}$ Carlie Driscoll ${ }^{2}$
}

To cite: Kaspar A, Pifeleti S, Driscoll C. Knowledge and attitudes of university health students in the Pacific Islands towards childhood hearing Ioss and hearing services: a Samoan survey study protocol. BMJ Paediatrics Open 2021;5:e000998. doi:10.1136/ bmjpo-2020-000998

Received 20 December 2020 Revised 14 February 2021 Accepted 21 February 2021
Check for updates

(C) Author(s) (or their employer(s)) 2021. Re-use permitted under CC BY-NC. No commercial re-use. See rights and permissions. Published by BMJ.

${ }^{1}$ ENT Department, Tupua Tamasese Meaole Hospital, Apia, Samoa

${ }^{2}$ Hearing Research Unit for Children, University of Queensland, Brisbane, Queensland, Australia

Correspondence to Dr Annette Kaspar; annette. kaspar@gmail.com

\section{ABSTRACT}

Introduction The Pacific Islands have among the highest rates of ear disease and hearing loss in the world, especially among children. Given that Ear, Nose and Throat (ENT)/audiology specialists are limited in the region, the successful implementation of ear and hearing health services will depend on the participation and support of the wider community of health professionals. There are currently no studies from the Pacific Islands investigating the knowledge and attitudes of either health students or health professionals towards childhood hearing loss and hearing services.

Methods and analysis Survey of medical and nursing students $(n=370)$ currently enrolled at the National University of Samoa. Students will independently and anonymously complete a 10-item questionnaire. The questions assess attitudes to childhood hearing loss (two questions), knowledge of aetiology of childhood hearing loss (three questions), and knowledge of identification and interventions for children with hearing loss (five questions). Responses are based on a 5-point Likert scale (Strongly agree/Agree/Neutral/Disagree/Strongly disagree).

Discussion/conclusion We publish these protocols to facilitate similar studies in other low-income and middleincome countries, and especially among our Pacific Island neighbours.

\section{INTRODUCTION}

According to the WHO, the Pacific Island region has among the highest rates of ear disease and hearing loss in the world. ${ }^{1}$ Otitis media (OM) and infectious diseases that are preventable through immunisations are the leading causes of hearing disorders in children. $^{1-5}$ The high prevalence of OM in the Pacific region is generally attributed to multiple factors which include low socioeconomic status and living conditions, challenges accessing medical care, malnutrition and frequent upper respiratory tract infections. ${ }^{67}$ Where immunisation coverage rates are below target levels, children are at risk of developing permanent hearing loss following

\section{What is already known on this topic?}

Pacific Island region has among the highest global rates of ear disease and hearing loss.

- Ear and hearing health specialists are virtually nonexistent in the Pacific Islands.

- A public health approach is recommended to reduce the burden of preventable ear disease and hearing loss.

\section{What this study hopes to add?}

- Assessment of Samoan university health student knowledge and attitudes to childhood ear disease and hearing loss.

- Information to guide translational/evidence-based university curricula and public/primary health policies in the Pacific Islands.

- Contribution to the limited research on ear and hearing health in the Pacific Islands.

measles or meningitis disease. ${ }^{8} 9$ A public health approach is therefore advocated to improve the ear and hearing health of the paediatric population in the Pacific Islands. ${ }^{10}$

Ear, Nose and Throat (ENT) specialists and audiologists are scarce in the Pacific Islands. ${ }^{1112}$ At the time of writing, the Pacific ENT and Audiology Group reports three ENT specialists (including author SP) and one audiologist (author AK) living permanently in the Pacific Islands. Alongside the public health approach, efforts to improve early identification and intervention for children with ear disease and hearing loss should be possible at this time through the wider community of health professionals. This aligns with the current WHO advocacy of task sharing for primary ear and hearing healthcare. There are currently no studies from any Pacific Island country investigating the 
knowledge and attitudes of health professionals towards hearing disorders.

The support of health professionals is essential to the successful implementation of ear and hearing health services for children. Studies from the Pacific Islands report that $\mathrm{OM}$ begins early in life, and may progress to life-threatening complications if poorly managed. ${ }^{513} 14$ Studies from other low-/middle-income countries (LMICs) provide evidence that timely referral to the ENT clinic or audiology department by other health professionals requires improvement. ${ }^{15} 16$ As well as increasing the risk of life-threatening complications from untreated OM, it is well documented that unidentified hearing loss in children negatively impacts on speech, language and cognitive development. To facilitate best possible clinical care, an assessment of health professional knowledge and support of ENT/audiology services is therefore required. There is currently goodwill and momentum among key Pacific Island stakeholders to address the public health threat of OM. ${ }^{17}$

A review of the literature found four studies that surveyed health professionals and medical students in LMICs regarding their awareness of infant hearing health and services. ${ }^{18-21}$ The studies revealed overwhelming peer support for ENT/audiology professionals. Although there was room for improvement on knowledge of aetiology factors of childhood hearing loss, a common theme throughout the studies was that parental concern is always a reason to refer a child for ENT/audiology assessment. ${ }^{22}$

ENT and audiology services are limited but evolving in Samoa, a Polynesian nation of the Pacific Islands. There is only ENT Department in Samoa, and it is located in the capital city Apia at the Tupua Tamasese Meaole Hospital, the national government hospital funded by the Ministry of Health. Children may attend the ENT Department free of charge, and although referrals from health or education professionals are accepted, no formal referral is required. Children aged $0-14$ years currently represent $32.72 \% \quad(\mathrm{n}=64717)$ of the total Samoan population $(\mathrm{N}=195864)$. Given that only $18.2 \%$ of the Samoan population resides in urban settings, task sharing of ear and health services with our medical colleagues should begin to bridge the gap that currently exists between the urban and rural/remote population.

The first author is a public health, development and research audiologist specialising in the Pacific Islands (AK). The second author (SP) is a general/ENT surgeon, the acting head of the ENT department, and a key education partner for students attending the National University of Samoa (NUS) Schools of Medicine and Nursing. This presents an excellent opportunity to investigate the knowledge and attitudes of Samoan medical and nursing students, our future health professionals, towards ear and hearing health. Although the NUS was established in 1984, the School of Medicine was officially launched in 2014 under the Faculty of Health Sciences. As the first round of students prepares to graduate, the school is reviewing their curriculum, and has expressed a keen interest to expand the course content on ear and hearing health.

The aim of the present study is to assess the current knowledge of health students in Samoa to childhood hearing loss and hearing services. The results of the study should provide evidence for professional support of the evolving ENT and audiology clinic, as well as guide the development of NUS health student curriculum to include this specialist field. This would be a step towards the successful implementation of ENT/audiology services in Samoa, as well as reducing the burden of ear and hearing health disease in the country. This first study will be used as a baseline to monitor the effectiveness of any initiatives that follow the study. There are no previous studies of this kind from the Pacific Islands, and the results of this study may serve as a model for other Pacific Island nations.

Our aim in publishing this study protocol is to facilitate similar studies of this kind, especially among our Pacific Island neighbours. Given the scarcity of ear and hearing health specialists in the Pacific Islands, the enhanced integration of ENT/audiology curricula into the current university courses should make a positive contribution to reducing the burden of ear disease and hearing loss in the region.

\section{METHODS AND ANALYSIS}

\section{Patient and public involvement}

This study is part of a larger project entitled 'Development of ENT/Audiology services in Samoa'. Other studies (1) examine caregiver knowledge and attitudes to childhood ear disease and hearing loss, and (2) clinical outcomes of ear and hearing assessments among different childhood age groups. The proposed study addresses the referral pathway between primary healthcare physician and specialist ENT/audiology care.

\section{Planning and preparation \\ Personnel}

The study will be led by the research audiologist (first author AK) and the head of ENT clinic (SP). A representative from the School of Health (NUS) will assist with the data collection. Data analysis will be performed in collaboration with the Hearing Research Unit for Children at the University of Queensland (Australia) and includes consultation with a biostatistician (CD).

\section{Design}

The study will use a convenience sampling design. Given time and resource restrictions, this is the most effective study design.

Setting

The study will take place at the NUS (Apia).

\section{Participants}

The study population will consist of medical and nursing students $(\mathrm{N}=370)$ currently enrolled at the NUS. There 
Table 1 Knowledge and attitudes of medical and nursing students to childhood hearing loss and hearing services in Samoa Study Questionnaire

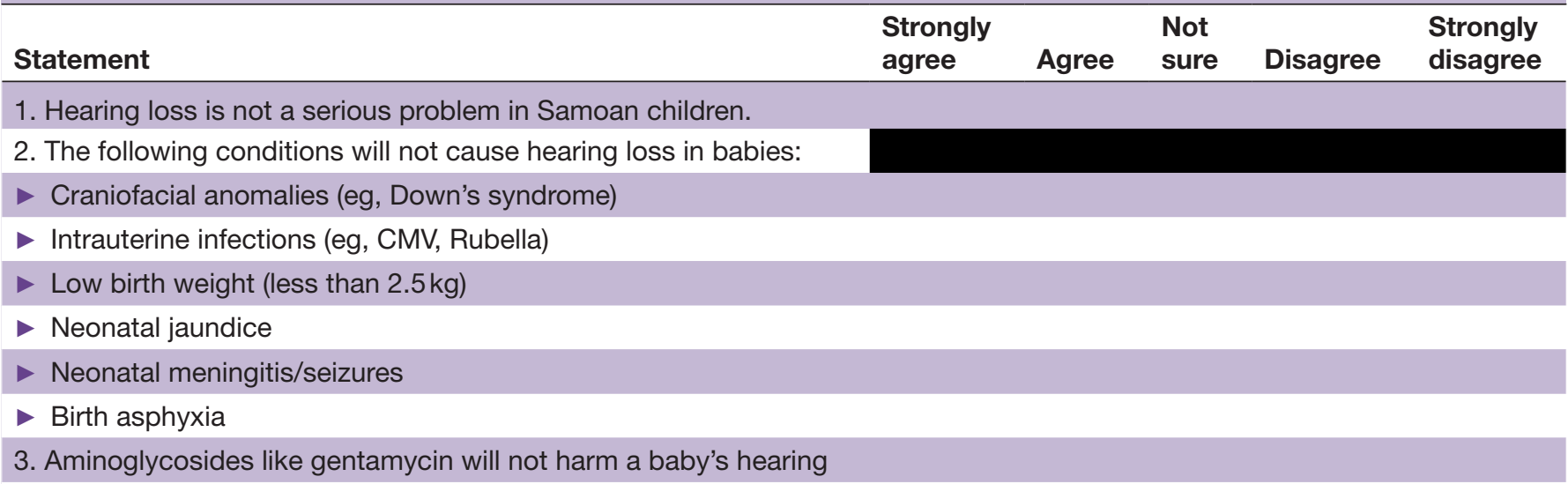

4. A mother's exposure to loud noise in pregnancy will not affect a baby's hearing

5. Babies with hearing loss cannot be accurately detected at birth

6. Babies with hearing loss cannot be helped until they are older

7. Hearing aids are unsuitable for babies

8. Parental suspicion of hearing loss should not influence a clinical opinion

9. Babies can wait until they are older before testing for hearing loss

10. Hearing loss is not that important because it does not kill

are 6-year levels for medical students $(\mathrm{N}=70)$, and 3-year levels for nursing students $(\mathrm{N}=300)$. Health students over 18 years of age attending class on the days of data collection will be eligible to participate in the study.

\section{Assessment tool: questionnaire}

The assessment tool will be the same questionnaire that was used in the original study performed in Nigeria ${ }^{19}$ (table 1). We considered the adaptation of this questionnaire that was used in Mexico, ${ }^{21}$ but judged that the original was better suited to our context. The ENT clinic team reviewed the 10 -item questionnaire and decided that the only modification required was amending 'Nigerian children' to 'Samoan children'. The research team judged it appropriate to administer the questionnaire in English, and this was approved by the Government of Samoa Health Research Ethics Committee.

The questionnaire will be completed independently and anonymously by the participants. The response required is on a 5-point Likert scale: 'Strongly agree', 'Agree', 'Neutral', 'Disagree' and 'Strongly disagree'.

\section{Equipment and consumables}

All requirements, as listed next, will be contained within a simple plastic folder for utilisation by the research team:

- Information sheet for participants (English) - knowledge and attitudes of medical and nursing students to childhood hearing loss and hearing services in Samoa.
- Group participant consent form (English)—knowledge and attitudes of medical and nursing students to childhood hearing loss and hearing services in Samoa

- Questionnaire (English) — knowledge and attitudes of medical and nursing students to childhood hearing loss and hearing services in Samoa.

- 'Ballot Box' for completed questionnaires.

- Staff member business cards with contact details.

- Pens.

N.B. To reduce the cost of consumables, a laminated copy of the information sheet and five non-laminated copies only will be in the study folder (information sheets to be kept by individuals only if requested). To further reduce the cost of consumables, each participant will sign a group consent form as they return their completed questionnaire.

\section{Conducting the study}

The recommended work flow on the days of data collection is summarised in figure 1.

\section{Recruitment of participants}

On the day(s) of data collection, the research team will attend a medical or nursing student class at NUS as arranged with the NUS representative. Half an hour before the class is due to end, the research team will be invited by the class lecturer to make an announcement about the study to the class. The announcement will introduce the members of the study team, and then describe the aims of the project (participant information sheet) 

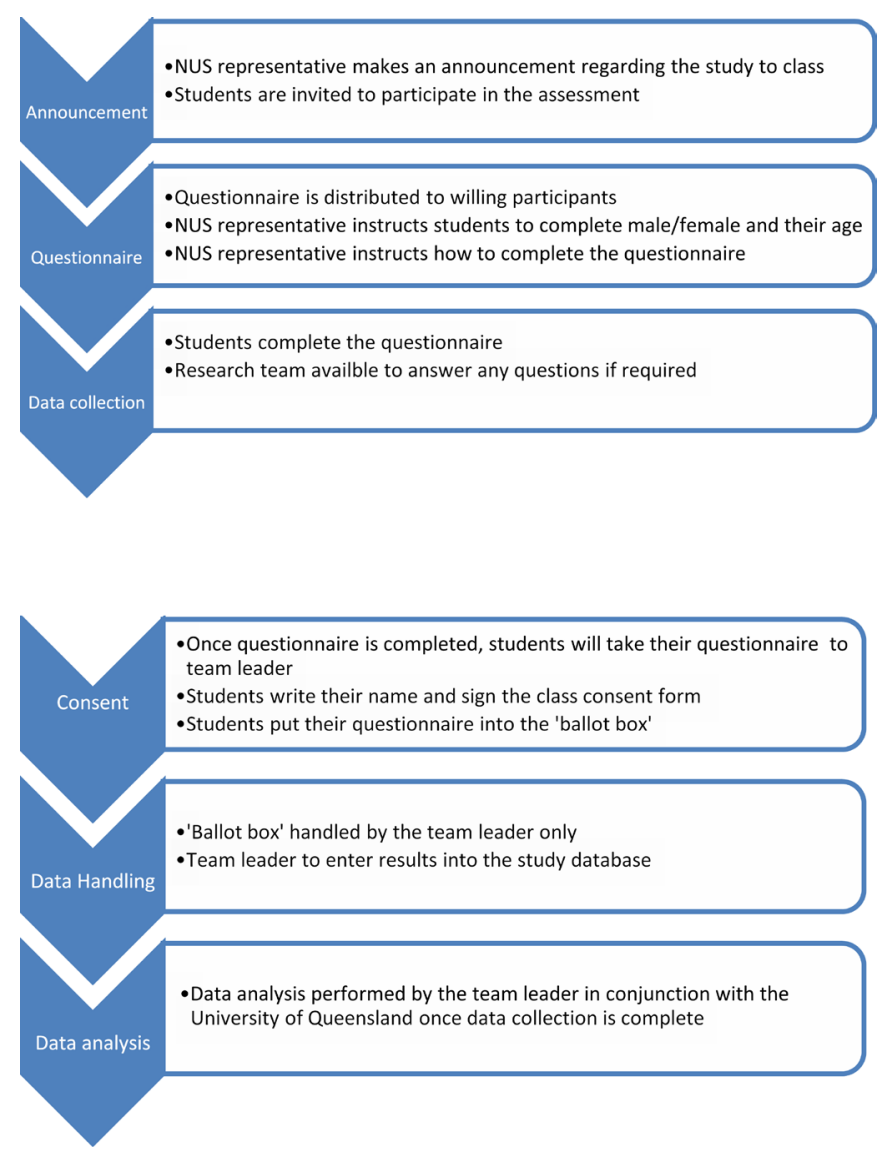

Figure 1 Knowledge and attitudes of medical and nursing students to childhood hearing loss and hearing services in Samoa study work flow chart. NUS, National University of Samoa.

and the participant requirements (including signing the group participant consent form when completed questionnaire is returned to the research team). The students will then be invited to participate in the study. The study questionnaire will be distributed to all willing participants.

\section{Administration of questionnaire Demographic information}

The NUS representative will instruct the participants to complete demographic information on their questionnaire as follows:

- Circle male/female as appropriate.

- Age (in years).

N.B. The completed questionnaires will be bundled together and labelled by the research team with the class name (ie, year 1 medical students).

\section{Questionnaire}

The questionnaire will be completed individually and anonymously by each student. They will be instructed to respond to each statement on the questionnaire with 'Strongly agree', 'Agree', 'Not sure', 'Disagree', or 'Strongly Disagree', and to mark their response in the appropriate box with an ' $\mathrm{X}$ '.
On completion of the questionnaire, the participants will be instructed to sign the Group Consent Form, and to place their completed questionnaire into the "ballot box' provided by the research team.

\section{Data handling and analysis}

All data handling and analysis will be done by the team leader. The ballot box with the completed questionnaires will remain closed until data entry is performed by the team leader at the ENT clinic. Each questionnaire will be identified by a number written in the top right-hand corner, and this will correspond to the order in which the questionnaires are removed from the ballot box (ie, 1, 2, 3 , etc). The team leader will enter all data into the SPSS Statistical Package Software in a file titled "Knowledge and Attitudes of Medical and Nursing Students to Childhood Hearing Loss and Hearing Services in Samoa."

The team leader will analyse the data to provide the following information:

\section{Demographic information}

A descriptive analysis will be performed for the total number of participants, number/proportion of males versus females, and number/proportion of medical versus nursing students according to study year level. The analysis will include age range (mean and SD) for the total number of participants, as well as for each subgroup. Results will be presented in tabulated form using a cross analysis.

\section{Analysis of variables}

For each item of the questionnaire, the number of participants answering 'Strongly agree', 'Agree', 'Not sure', 'Disagree', or 'Strongly disagree' will be tallied and tabulated. Spearman's correlation coefficient will be used to identify any significant differences in response proportions between groups.

\section{DISCUSSION/CONCLUSION}

The results of this study will be prepared as a research paper for submission to a peer-reviewed journal. The study from Nigeria was published in paediatric rehabilitation. Other options under consideration at this time include the International Journal of Pediatric Otorhinolaryngology and the International Journal of Audiology. The published paper will be also be delivered to the Government of Samoa Ministry of Health and the NUS, including an action plan for any recommendations to the current university course curricula.

The Ministry of Health and NUS will prepare a short media release about the study and the results. This will be done in collaboration with the Government of Samoa to ensure information is appropriate and receives maximum population coverage. The aim of the media release is (1) to promote the role of the wider health professional community in ear and hearing health, (2) to increase community awareness of the adverse effects 
of ear disease and associated hearing loss on childhood development, and (3) to encourage timely attendance at health services for treatment of ear disease.

We include the participant information sheet and group participant consent form as additional files with this publication to further facilitate similar studies of this kind, especially among our Pacific Island neighbours.

Contributors AK wrote the first draft of the manuscript, and coauthors SP and CD provided feedback. AK made revisions as appropriate, and coauthors SP and CD approved the final manuscript.

Funding The authors have not declared a specific grant for this research from any funding agency in the public, commercial or not-for-profit sectors.

\section{Competing interests None declared.}

Patient and public involvement Patients and/or the public were not involved in the design, or conduct, or reporting, or dissemination plans of this research.

Patient consent for publication Not required.

Ethics approval This study was approved by the Government of Samoa Ministry of Health Ethical Research Committee and the University of Queensland Medical Ethics Research Committee (Approval No. 2020000255). Gatekeeper approval was obtained from the National University of Samoa.

Provenance and peer review Not commissioned; externally peer reviewed.

Data availability statement No data are available. All data relevant to the study will be included in the published journal article.

Open access This is an open access article distributed in accordance with the Creative Commons Attribution Non Commercial (CC BY-NC 4.0) license, which permits others to distribute, remix, adapt, build upon this work non-commercially, and license their derivative works on different terms, provided the original work is properly cited, appropriate credit is given, any changes made indicated, and the use is non-commercial. See: http://creativecommons.org/licenses/by-nc/4.0/.

ORCID iD

Annette Kaspar http://orcid.org/0000-0002-4035-3236

\section{REFERENCES}

1 WHO. Who global estimates on prevalence of hearing loss, 2018. Available: https://www.who.int/pbd/deafness/estimates/en/

2 Leach AJ, Homøe P, Chidziva C, et al. Panel 6: otitis media and associated hearing loss among disadvantaged populations and low to middle-income countries. Int J Pediatr Otorhinolaryngol 2020;130:109857.

3 Monasta L, Ronfani L, Marchetti F, et al. Burden of disease caused by otitis media: systematic review and global estimates. PLoS One 2012;7:e36226.
4 Mahadevan M, Navarro-Locsin G, Tan HKK, et al. A review of the burden of disease due to otitis media in the Asia-Pacific. Int $J$ Pediatr Otorhinolaryngol 2012;76:623-35.

5 Kaspar A, Kei J, Driscoll C, et al. Overview of a public health approach to pediatric hearing impairment in the Pacific Islands. Int $J$ Pediatr Otorhinolaryngol 2016;86:43-52.

6 WHO. Chronic suppurative otitis media: burden of illness and management options. Geneva: WHO, 2004. https://www.who.int/ $\mathrm{pbd} /$ publications/Chronicsuppurativeotitis_media.pdf?ua $=1$

7 WHO. Childhood hearing loss. strategies for prevention and care. Geneva: WHO, 2016. http://apps.who.int/iris/bitstream/handle/ 10665/204632/9789241510325_eng.pdf?sequence=1

8 Kaspar A, Pifeleti S. Brief overview of meningitis in the Pacific Islands, and implications for public health, clinical, and rehabilitation services: a call to action. J Glob Health.

9 Kaspar A, Pifeleti S, Whitfield BC. The measles emergency is over, but the crisis continues - a call to action for the Pacific Islands. $J$ Glob Health 2020;10:020301.

10 Kaspar A, Kei J, Driscoll C, et al. A public health approach to pediatric hearing impairment in the Pacific Islands. J Glob Health 2018:8:010302.

11 Sanders M, Houghton N, Dewes O, et al. Estimated prevalence of hearing loss and provision of hearing services in Pacific island nations. J Prim Health Care 2015;7:5-15.

12 Thorne PR, Holt EA, Nosa V, et al. Provision of hearing care in Pacific island countries and territories. Bull World Health Organ 2019;97:719-21.

13 Dubey SP, Larawin V. Complications of chronic suppurative otitis media and their management. Laryngoscope 2007;117:264-7.

14 Dubey SP, Larawin V, Molumi CP. Intracranial spread of chronic middle ear suppuration. Am J Otolaryngol 2010;31:73-7.

15 Jafari Z, Malayeri S, Ashayeri $\mathrm{H}$. The ages of suspicion, diagnosis, amplification, and intervention in deaf children. Int J Pediatr Otorhinolaryngol 2007;71:35-40.

16 Ozcebe E, Sevinc S, Belgin E. The ages of suspicion, identification, amplification and intervention in children with hearing loss. Int $J$ Pediatr Otorhinolaryngol 2005;69:1081-7.

17 Holt E, McCool J, Nosa V, et al. Development of an otitis media strategy in the Pacific: key informant perspectives. N Z Med $J$ 2018:131:69-76

18 Yerraguntla K, Ravi R, Gore S. Knowledge and attitude of pediatric hearing impairment among General physicians and medical interns in coastal Karnataka, India. Indian J Otol 2016;22:183-7.

19 Olusanya BO, Roberts AA. Physician education on infant hearing loss in a developing country. Pediatr Rehabil 2006:9:373-7.

20 Campos ACMde, Shirane HY, Takemoto PVA, et al. [Universal newborn hearing screening: knowledge of pediatricians and neonatologists in the city of Jundiaí, São Paulo, Brazil]. Braz J Otorhinolaryngol 2014;80:379-85.

21 López-Vázquez M, Berruecos P, Lopez LE, et al. Attitude and knowledge of hearing loss among medical doctors selected to initiate a residency in Mexico. Int J Audiol 2009;48:101-7.

22 Ravi R, Gunjawate DR, Yerraguntla K, et al. Systematic review of knowledge of, attitudes towards, and practices for newborn hearing screening among healthcare professionals. Int $J$ Pediatr Otorhinolaryngol 2018;104:138-44. 